\title{
Fast adaptive routing supporting mobile senders in Source Specific Multicast
}

\author{
Thomas C. Schmidt • Matthias Wählisch • Maik Wodarz
}

Published online: 15 October 2009

(C) Springer Science+Business Media, LLC 2009

\begin{abstract}
IP multicast deployment recently progresses, but group services often remain restricted to limited domains and fail to comply with route-optimizing mobility management of the next generation Internet. Source Specific Multicast (SSM) facilitates transparent inter-domain routing and is expected to globally disseminate to many users and services. However, mobility support for Source Specific Multicast is still known to be a major open problem. In this paper, we propose the Enhanced Tree Morphing (ETM) protocol for extending SSM routing to mobile multicast sources. The scheme dynamically adapts SSM forwarding states to sender mobility, and transforms (morphs) source specific distribution trees into new, optimal trees rooted at a relocated source. ETM is simple, robust and secure, while it admits superior performance in packet forwarding at a low signaling overhead. Extensive evaluations based on a full protocol implementation, and simulations based on real-world topology data are performed, granting full insight into the properties of packet loss and delay stretch, protocol convergence times and router state evolution during single and rapidly repeated handovers. In a constant bit rate scenario, an ETM source handover typically leads to a slightly increasing de-
\end{abstract}

T.C. Schmidt $(\varangle) \cdot$ M. Wählisch · M. Wodarz

Department Informatik, HAW Hamburg, Berliner Tor 7,

20099 Hamburg, Germany

e-mail: T.Schmidt@ieee.org

M. Wählisch

e-mail: Waehlisch@ieee.org

M. Wodarz

e-mail: Maik.Wodarz@informatik.haw-hamburg.de

M. Wählisch

Institut für Informatik, Freie Universität Berlin, Takustr. 9, 14195 Berlin, Germany lay of the first data packet, only. When operating on realistic network topologies, the protocol uniformly converges within less than $50 \mathrm{~ms}$, thereby sustaining robustness under rapid source movement at all speeds common to our mobile world. Further optimizations are identified for FMIPv6 and for multihomed nodes.

Keywords Mobile multicast routing $\cdot$ SSM $\cdot$ Source Mobility · Route optimization · Mobile IPv6 · FMIPv6 · Multihoming $\cdot$ Performance analysis

\section{Introduction}

Mobile communication today follows the trend to converge with networked applications to a common IP-centric world. Throughout this process, Internet services are expected to extend to mobility management in the near future. The virginal availability of a new, truly mobile IP enabled network layer [1] offers connectivity to nomadic users at roaming devices, while preserving communication sessions beyond IP subnet changes.

Since IPTV offers have become widely adopted by operators, the deployment of IP multicast has accelerated. Simplicity and cost efficiency, but also a universal availability at client sides, motivate new content distribution services to be established with the help of group communication on the Internet layer. Additional community-oriented applications like MMORPGs, collaborative environments and conferencing increase the demand for ubiquitously available group communication. Current multicast deployment, however, lacks transparency in two vital aspects. First, the traditional Internet approach of Any Source Multicast (ASM) [2] fails to provide a scalable, domain-transparent multicast routing, 
leading operators to restrict its deployment to walled gardens. Second, mobility has not been designed as a standard for a scalable, efficient multicast solution. Despite of more than ten research years, mobile multicast protocol development is still in a premature state [3].

Simple, transparent inter-domain multicast routing is introduced by the deployment-friendly, more selective Source Specific Multicast (SSM) model [4]. SSM is expected to globally disseminate to many users and services. In contrast to Any Source Multicast (ASM), optimal $(S, G)$ multicast source trees are constructed immediately from $(S, G)$ subscriptions at the client side, without utilizing network flooding or rendezvous points. Source addresses are to be acquired out of band, which an IPTV program guide or a SIP [5] session initiation in conferencing scenarios may facilitate [6]. However, mobility support for Source Specific Multicast is still known to be a major open problem.

This paper addresses the issue of mobile Source Specific Multicast routing on the network layer by presenting the Enhanced Tree Morphing (ETM), an optimized multicast routing protocol for transforming (morphing) source specific distribution trees into optimal trees rooted at a relocated source. The ETM protocol is simple, robust and secure, and admits superior performance in packet forwarding. As will be shown in the remaining paper, ETM adheres to real-time compliant performance. Extensive evaluations based on a full protocol implementation operating on real-world topology data are performed, granting full insight into the properties of packet loss and delay stretch, protocol convergence times and router state evolution during single and rapidly repeated handovers. The scheme can be implemented with little overhead, and by applying minimal modifications to current standard protocols for unicast mobility management.

This paper is organized as follows. We first introduce and discuss the mobile multimedia group conferencing problem and related work. In Sect. 3, we present the core protocol. A thorough evaluation follows in Sect. 4. Finally, Sect. 6 is dedicated to a conclusion and an outlook.

\section{The mobile Source Specific Multicast problem and related work}

\subsection{Problem statement}

A mobile multicast sender will face the problem of enabling a continuous forwarding of data to its group of receivers, while it undergoes roaming and network layer handovers. Its mobility protocol should facilitate a seamless transmission service and at the same time preserve transparency with respect to network and address changes at the receiver side. Multicast listener applications are frequently source address aware. A mobile multicast source consequently must meet address transparency at two layers: To comply with RPF checks, it has to use an address within the IPv6 basic header's source field, which is in topological agreement with the multicast distribution tree controlled by routers. For application transparency, the logical node identifier, commonly the Home Address, must be presented as the packet source address to the transport layer at the receivers. This aspect of address duality is inherited from unicast mobility management.

At the complementary side, network routing must comply with sender movement without having network functionality compromised. It should realize native forwarding whenever possible to preserve its resources, but needs to ensure routing convergence even under a rapid movement of the sender. Mobility support for multicast sources at the network layer thus poses a significant challenge to the infrastructure. An SSM node submitting data to a group of receivers defines the root of a source specific shortest path tree (SPT), distributing data towards its receivers. Native forwarding along source-specific delivery trees will be bound to the topological network address of the source, as kept under surveillance by reverse path forwarding (RPF) checks. SSM source filters apply orthogonally to the Home Address for source identification. Address duality management is thus required at the routing level for mobile SSM.

A mobile multicast source moving to a new subnetwork is only able to either inject data into a previously established delivery tree, or to (re-) initiate the construction of a multicast distribution tree compliant to its new location. In the latter case, the mobile sender will have to trigger receivers, but needs to proceed without controlling the new tree development, as it operates decoupled from receiver and router operations. Lack of synchronization poses the threat for a source of loosing contact to its receivers.

\subsection{Related work}

More than ten years ago, at the early design-time of Internet mobility [7], initial light was shed on IP multicast extensions for mobile hosts. Xylomenos \& Polyzos [8] introduced home subscription and remote subscription for mobile nodes as the two basic mechanisms that remain compliant to generic multicast routing. While remote subscription cannot be applied to mobile SSM senders, any mobile multicast source (MS) may transparently transmit data using bi-directional tunneling through its home agent. Adopted as a minimal standard in MIPv6 [1], bi-directional tunneling completely hides mobility operations from multicast routing at the price of triangular paths and extensive encapsulation.

This concept of static distribution trees has been extended to take advantage of the multicast routing infrastructure. Following a shared tree approach, Romdhani et al. [9] propose to replace Home Agents in their role as mobility 
anchors by the Rendezvous Points of PIM-SM [10]. Mobile senders tunnel their data to these "Mobility-aware Rendezvous Points" (MRPs), whence in restriction to a single domain, this scheme is equivalent to the bi-directional tunneling. Focusing on interdomain mobile multicast, the authors design a tunnel- or SSM-based distribution backbone between MRPs. In attaching to a regional MRP, a mobile node thus weakens performance degradations inherited from triangular routing via a single Home Agent.

Statically anchored multicast trees grant simplicity and route stability to mobile sources, but cannot provide optimal routing paths. To overcome this deficit, several authors propose to construct a completely new distribution tree after the movement of a mobile source. Such schemes have to rely on client notification for triggering router state establishment anew. At the same time, they need to preserve address transparency to clients. To account for the latter, Thaler [11] proposes to employ binding caches and to obtain source address transparency analogous to MIPv6 unicast communication. Initial session announcements and changes of source addresses are to be distributed periodically to clients via an additional multicast control tree centered at the home agent. Source-tree handovers are then activated on listener requests. Jelger and Noel [12] suggest handover improvements by employing anchor points within the source networks. Anchors are spanned by tunnels for supporting a continuous data reception during client-initiated handovers.

The reconstruction of a completely new distribution tree may be complex, costly and slow, even though in many realistic handover scenarios a significant coincidence in states of the previous and next multicast shortest path tree can be observed [13]. From this perspective, algorithms for reusing major portions of existing multicast forwarding states, while modifying minor parts of distribution trees, have been designed. Such schemes aim to continuously serve for data transmission of mobile sources, and at the same time minimize routing update cost. In the ASM case of DVMRP routing, [14] propose an algorithm to extend the root of a given delivery tree to incorporate a new source location. Vida et al. [15] propose a recursive scheme of loose unicast source routes with branch points, based on their Multicast Hop-byHop routing protocol [16]. M-HBH optimizes shortest paths trees for a moving source on the path between the source and the first branching point. O'Neill [17] suggests a scheme to overcome RPF-check failures originating from multicast source address changes, by introducing an extended routing information, which accompanies data in a Hop-by-Hop option header.

A routing protocol adaptive to SSM source mobility, the Tree Morphing, has been introduced by the authors in $[18,19]$. A mobile multicast source (MS) away from home will transmit unencapsulated data to a group, using its current $\mathrm{CoA}$ on the Internet layer, but HoA on the application layer, which is carried in extension headers like in MIPv6. In extension to unicast routing, though, the entire Internet layer, i.e. routers included, will be aware of the permanent HoA. Maintaining binding-cache-like address pairs in router states will enable all multicast routers to simultaneously identify $(H o A, G)$-based group membership and $(C o A, G)$-based tree topology. When moving to a new point of attachment, the MS will alter its address from previous $\mathrm{CoA}(\mathrm{pCoA})$ to new $\mathrm{CoA}(\mathrm{nCoA})$ and eventually change from its previous Designated multicast Router (pDR) to a next Designated Router (nDR). Subsequent to handover it will immediately continue to deliver data along an extension of its previous source tree using source routing from $\mathrm{nDR}$ to pDR. All routers along this path will learn the new CoA of MS and implement appropriate forwarding states. Routers on this extended tree use RPF checks to discover potential short cuts. In the absence of short cuts, routers re-use those parts of the previous delivery tree, which coincide with the new shortest path tree. Only branches of the new shortest path tree, which have not previously been established, need to be constructed. In this way, the previous shortest path tree will be morphed into a next shortest path tree.

Partially similar, Lee et al. [20] introduce a state update mechanism for mobile multicast sources that targets at reusing major parts of established multicast trees. The authors start from initially established distribution states centered at the mobile source's home agent. A mobile leaving its home network will signal a multicast forwarding state update on the path to its home agent, and subsequently, distribution states according to the mobile source's new CoA are implemented along the previous distribution tree. Multicast data then is intended to natively flow in triangular routes via the elongated and updated tree centered at the home agent. Unfortunately the authors do not address the problem of RPF check failures in their paper.

\subsection{Discussion}

Bi-directional tunneling is considered only a minimal solution, operational without explicit multicast mobility support. It introduces considerable overheads from triangular routing and wide-spanned encapsulation. Handover assistance via mobility-aware rendezvous points relies on triangular routing and tunneling, as well, and in addition re-introduces RPs, which are not native to SSM routing.

Receiver oriented tree reconstructions in SSM suffer from the problem of receivers being unsynchronized to source handovers. The adoption of a persistent control tree will resolve this issue, but introduces the overhead of an additional multicast tree spanning all receivers. In addition, handover messages distributed via a control tree will require a roundtrip signaling between source and receivers and consequently may be slow in widespread topologies. 
The MSSMSv6 approach [12] will expedite the handover process, but fails to cope without client synchronization. In the case of rapid movement, the authors are leaving the source with an unlimited number of 'historic' delivery trees to be fed simultaneously.

Both, bi-directional tunneling and tree reconstruction schemes have been analysed in simulations [19]. Large fluctuations of packet delays were identified with delay stretches growing up to a factor of 3 . For the tree reconstruction schemes, protocol convergence times likewise fluctuate significantly with Internet topologies, and state maintenance cost grows linearly in the number of receivers. Results evidently urged the need for routing enhancements, delivering better performance to the users and reducing mobilityinduced retroactivity in the network.

Adaptive tree approaches offer a solution space that addresses both, seamless handovers and sustained receiver contact. In addition, they can take advantage of limited mobility-related changes in the shapes of multicast distribution trees that can be observed [13]. They need to tackle the problem of RPF-incongruity, though, whenever intermediate forwarding states do not fall onto a valid SPT. The Tree Morphing protocol complies well with these advantages and constraints, but suffers from two significant drawbacks. At first, it relies on an initial source routing of packets, which appears elegant from the perspective of protocol design, but faces severe deployment objections from the perspective of operators. Source routing is considered a general security threat and frequently defeated. At second, extensive simulation studies revealed not only an undesirable delay stretch in initial packet delivery, but a realistic likelihood that the router convergence process will cause packet loss for those packets, initially routed via the previous designated router. For these reasons, we will present an enhanced version of the protocol in the following section that operates without source routing, omits packet loss and attains expedited forwarding and protocol convergence.

\section{The Enhanced Tree Morphing protocol}

The Enhanced Tree Morphing protocol (ETM) defines mobility management extensions to regular SSM routing. These are not bound to a specific multicast routing protocol, but only require join and prune functions for source-specific states at outgoing interfaces. For simplicity, our presentation follows the concepts of PIM-SSM [10], the primary SSM routing protocol available today.

\subsection{Overview}

ETM mobility extensions must provide address duality treatment at the receiver and at the router level. Therefore, a mobile multicast source (MS) away from home will transmit unencapsulated data to a group using its current $\mathrm{CoA}$ on the Internet layer, but its HoA on the application layer. The latter is carried in a mobility destination option header as defined in MIPv6. In extension to unicast routing, though, the multicast routing layer will be aware of the permanent HoA by maintaining $(C o A, H o A, G)$ address triples in router states.

A seamless handover management requires multicast transmission to continue immediately after IP layer reassociation has been obtained from the unicast mobility protocol in operations, e.g., MIPv6 [1], Mobile IPv6 Fast Handovers (FMIPv6) [21], or Hierarchical Mobile IPv6 (HMIPv6) [22]. When moving to a new point of attachment, the MS will alter its previous $\mathrm{CoA}(\mathrm{pCoA})$ to new $\mathrm{CoA}$ (nCoA), and eventually change from its previous Designated multicast Router (pDR) to a next Designated Router (nDR). Subsequent to handover, it will immediately inject forwarding states on the route from nDR to pDR by submitting an authenticated unicast state update packet (cf. Sect. 3.2) to pDR. As a result, the previous delivery tree will be elongated by $(n C o A, H o A, G)$ states as shown in Fig. 1(a). MS then continues to deliver data along this extended previous source tree. Delivery is done by including state update message headers in the first data packet(s). All routers along the previous delivery tree will thereby learn MS's new CoA and add appropriate forwarding states.

Routers participating in the ETM protocol will provide two additional functions: The STATE INJECTION Algorithm and the Extended Forwarding AlgoRITHM as shown below. State injection is triggered by a state update messages with a Hop-by-Hop router alert. The new ( $n C o A, H o A, G)$ state is implemented hopwise on the forwarding interfaces of the previous tree. Previous $(p C o A, H o A, G)$-states are kept, whenever the update packet has been received on a topological incorrect interface. In this way, routers may maintain several versions of the distribution tree until the protocol converges to one new SPT. In detail this algorithm runs as follows:

\section{STATE InJECTION ALGORITHM}

$\triangleright$ Upon receiving an $(n C o A, H o A, G)$

$\triangleright$ state update for multicast group $G$

1 for all $(\cdot, H o A, G)$ Forwarding Interfaces

2 do if $(\mathrm{RPF}-\mathrm{CHECK}(n C o A)=$ TRUE $)$

3 then REPLACE all $(\cdot, H o A, G)$-states by $(n C o A, H o A, G)$

4 5 else $\operatorname{ADD}(n C o A, H o A, G)$-state INIT TREE_OPTIMIZATION 


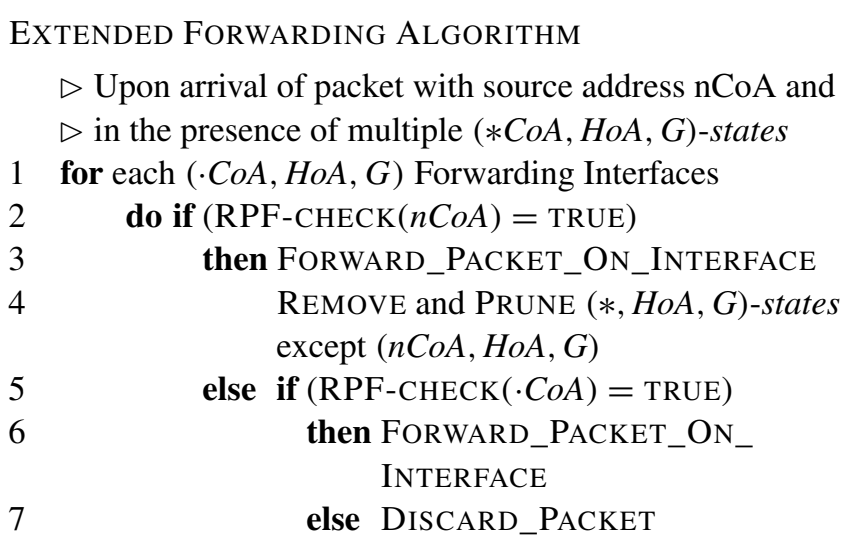

After an update has been processed, the packet is passed on according to the EXTENDED FORWARDING ALGORITHM described below. At this stage, the delivery tree does not need to be optimal and packets may fail at standard RPF checks. To prevent unwanted discarding, incoming packets need to be accepted from any interface that holds a $(\cdot, H o A, G)$-state, ${ }^{1}$ i.e., a topological member of the current or some previous distribution tree. Up until tree optimization has completed locally, this requires to apply the extended forwarding which simultaneously accounts for all source address states $(\cdot, H o A, G)$. Packets thereby will be forwarded along some $(C O A, H o A, G)$ tree, provided they arrived at the topologically correct interface for this particular $C o A$.

Any router will detect suboptimal routes from packets arriving at a topological incorrect interface (w.r.t. the source address of the packet). It will dynamically create an optimal shortest path tree, similar to the PIM-SM switching mechanism from a shared-tree to a shortest-path tree. After receiving a multicast packet for group $(\cdot, H o A, G)$ with source address $n C O A$ and RPF failure, a router will immediately submit a join to $(n C o A, G)$. The underlying SSM routing protocol will initiate the construction of a shortest path source specific branch. The router will learn about its completion by $(n C o A, H o A, G)$ traffic arriving at the correct interface and thereafter will prune $(*, H o A, G)$ on all incoming interfaces corresponding to previous $C o A$ addresses. ${ }^{2}$ Figure 1(b) visualizes such an intermediate morphing state.

A tree will be locally optimal, as soon as packets arrive at the topological correct interface, and states have converged

\footnotetext{
${ }^{1}$ Further on we will denote "some state with group address $G$ and home address $H o A$ " by $(\cdot, H o A, G)$, whereas $(*, H o A, G)$ stands for all such states.

${ }^{2}$ It should be noted that state update order maintenance is omitted for the sake of simplicity. In cases of very rapid movement, in which the handover frequency is higher than the protocol convergence rate, this may lead to unwanted cuts of tree branches. However, evaluations in this paper show that protocol convergence is significantly faster than handovers from vehicular movement (cf. Sect. 4.5).
}

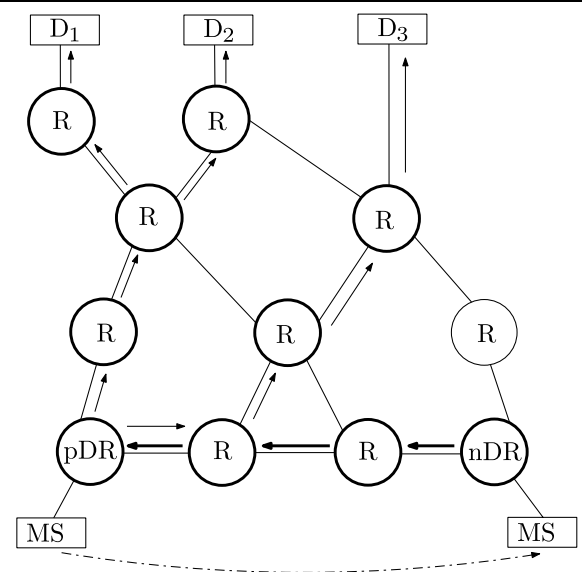

(a) Initial tree with elongation

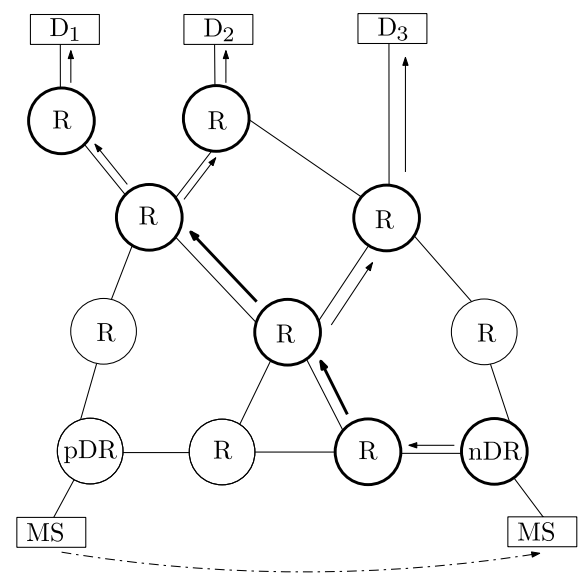

(b) Inter-tree morphing

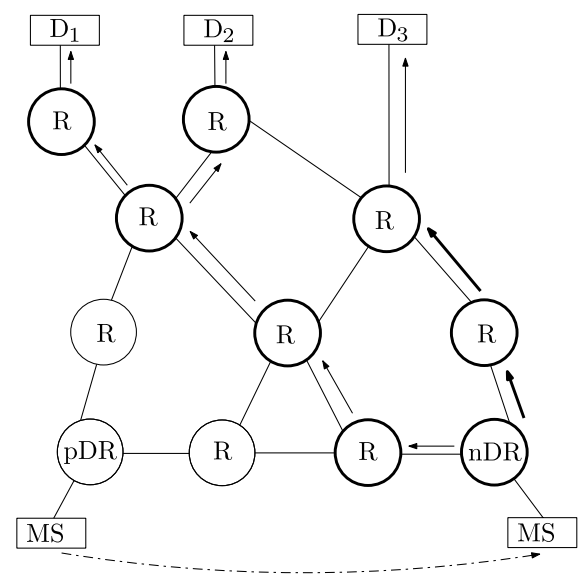

(c) Converged new tree

Fig. 1 Tree morphing states neighboring to the source with receiver domains $D_{i}$

to $(n C o A, H o A, G)$. The details of this extended forwarding algorithm read:

In applying this forwarding algorithm, the delivery tree thus will not only transport intermediate detouring packets, but will continuously degenerate branches dispensable due to optimization incidences. As soon as $(*, H o A, G)$ forwarding states have reduced to a single $(\cdot, H o A, G)$ entry, the 
router operations continue as defined by its standard multicast routing protocol without mobility extension.

Finally, state update packets will arrive at the receivers of the $(\cdot, H o A, G)$ SSM group. The mobile IPv6 stack will interpret the binding update and alter its multicast binding cache entry. Thereafter, the standard destination option header is processed and data is transparently passed at $(H o A, G)$-endpoints to the transport layer.

\subsection{Packet design}

The networking layer requires information of state changes, whenever a multicast source address changes after a Mobile IPv6 handover. Signaling is first needed as a unicast message from $\mathrm{pDR}$ to $\mathrm{nDR}$ at the initial tree elongation phase, and second as a multicast state update to all routers on the previous distribution tree. The required information subsumes the group address, the home address and the care-of address, as well as proof of authentication. Updates bear the common risks of impersonation and theft of service in performing redirects on a global scale, and hence require a cryptographically strong authentication. The corresponding security issues are common to all approaches for mobile multicast sources, and have been recently combined in a joint approach called AuthoCast [23].

The unicast state update message can be composed of several IPv6 and mobility headers and there is no need to define a new protocol from scratch. A Router Alert Option inserted in a Hop-by-Hop Option Header [24] will instruct routers to further inspect packet headers and ensures that updates are interpreted by all routers along the paths. Additionally, regular CGA-authenticated Binding Updates [25] will carry the required information except for the group address. The latter may be inserted into the Binding Update Message as an additional Group Option, defined in analogy to the Alternate CoA address option [1]. The unicast update message is visualized in Fig. 2.

Multicast signaling is implemented on the network layer by inserting additional headers into the data packets. State update information including the group address are part of the autonomously verifiable header chain for Binding Updates as displayed in Fig. 3, and can be extracted from Binding Update messages sent by mobiles to correspondent end nodes subsequent to every unicast handover.

The complete ETM signaling is thus built by chaining IPv6 extension headers, to be sent in the initial unicast state update message, as well as for piggy-backing with the first multicast data packet(s). It is worth noting that only one state update message per handover is required at the routing infrastructure. To compensate for packet loss, updates may be repeated without significantly increasing processing overheads. Following a sequence identifier, routers will process updates only once. For a detailed discussion on security aspects and overheads we refer to [23].

\subsection{ETM finite state machine}

The finite state machine of the ETM protocol is derived of the PIM-SSM [10] state machine. The states No Info (NI), Join (J) and Prune Pending (PP) interact as in standard PIMSSM. The Join state is augmented by an ETM state, which represents the ETM router conditions during mobility management and prior to protocol convergence. Its characteristic lies in a state splitting initiated from state update messages, which in the event of rapid movement may be received multiple times prior to convergence. This is realized via a state counter and allows for a joined treatment of the correlated $(\cdot, H o A, G)$ stated within routers. The FSM has been used to verify the protocol formally.

\section{Protocol evaluation}

To evaluate the ETM behavior in detail, we implemented the full protocol, i.e., the corresponding functions of routers, sources and receivers within the network simulator platform OMNeT++ 3.3 [26]. We extended the IPv6Suite, which itself is based on the INET framework and already includes MIPv6 protocol operations. Investigations grant full insight into the properties of packet loss and delay stretch, protocol convergence times and router state evolution during single and rapidly repeated handovers.
Fig. 2 ETM IPv6 message for authenticated unicast state updates of mobile multicast sources

\begin{tabular}{|c|c|c|c|c|c|}
\hline IPv6 Header & $\begin{array}{l}\text { Hop-by-Hop } \\
\text { Option Header }\end{array}$ & $\begin{array}{c}\text { Destination } \\
\text { Options Header }\end{array}$ & & jility Header & \\
\hline $\begin{array}{l}\text { Src: } \mathrm{CoA} \\
\text { Dst: pDR }\end{array}$ & Router Alert Option & $\begin{array}{l}\text { Home Address } \\
\text { Option } \\
\text { HomeAddr. HoA }\end{array}$ & $\begin{array}{l}\text { Binding Update } \\
\text { Message } \\
\text { Group Option: G }\end{array}$ & $\begin{array}{c}\text { CGA } \\
\text { Parameter } \\
\text { Option }\end{array}$ & $\begin{array}{c}\text { CGA } \\
\text { Signature } \\
\text { Option }\end{array}$ \\
\hline
\end{tabular}

\begin{tabular}{|c|c|c|c|c|c|c|}
\hline \multicolumn{1}{c}{$\begin{array}{c}\text { Hop-by-Hop } \\
\text { IPv6 Header }\end{array}$} & $\begin{array}{c}\text { Destination } \\
\text { Option Header } \\
\text { Options Header }\end{array}$ & $\begin{array}{c}\text { Upper Layer } \\
\text { Header + Data }\end{array}$ \\
\hline $\begin{array}{c}\text { Src: CoA } \\
\text { Dst: G }\end{array}$ & Router Alert Option & $\begin{array}{c}\text { Home Address } \\
\text { Option } \\
\text { HomeAddr. HoA }\end{array}$ & $\begin{array}{c}\text { Binding } \\
\text { Update } \\
\text { Message }\end{array}$ & $\begin{array}{c}\text { CGA } \\
\text { Parameter } \\
\text { Option }\end{array}$ & $\begin{array}{c}\text { CGA } \\
\text { Signature } \\
\text { Option }\end{array}$ & $\begin{array}{c}\text { Data } \\
\end{array}$ \\
\hline
\end{tabular}

Fig. 3 ETM IPv6 header sequence for authenticated multicast state updates of mobile multicast sources 


\subsection{Simulation scenarios and measurements}

We performed stochastic discrete event simulations, taking initial conditions of source handover events, receiver locations and topology data as varying input. The following different scenarios were chosen.

Firstly, we chose artificial topologies to explore the attainable relative network geometry in the vicinity of the source. Secondly, we evaluated a single receiver scenario within several realistic topologies obtained from real-world measurements. Evaluations were performed as a function of routing complexity defined by the total motion of the source. This scenario was then extended to include a varying number of receivers, giving rise to analyzing the scaling behavior w.r.t. receiver multiplicities. Finally, protocol performance was tested while the source performed iterated handovers at different speeds.

To approximate realistic scenarios, most protocol evaluation have been performed on the basis of real-world topologies. Network data must be considered critical, as key characteristics of multicast routing are dominated by the topology in large networks. For comparative reasons, we chose the ATT core network [27] as a large (154 core nodes), densely meshed single provider example. For multiple provider Internet data we extracted sub-samples of 154 and 1.540 core nodes from the "SCAN + Lucent" map [28] project, further on denoted as "Internet" topology.

In detail, the simulations proceeded as follows: The mobile source continuously submits (numbered and timestamped) packets at a constant bit rate of $15 \mathrm{~ms}$, while performing handovers from one 802.11 WLAN access point to another. Access points are directly connected to the designated routers. Link delays in our setting have been homogeneously chosen to be $10 \mathrm{~ms}$, layer 2 handover times in the WLAN last $100 \mathrm{~ms}$ on average.

Our analysis covers packet delay, loss and evolution of router states. From the latter, protocol convergence times could be extracted for every router. Measurements have been performed with the help of a monitoring function at routers and receivers, which accounts for the maximal delay stretch, i.e., the ratio taken of the slowest packet, delivered during handoff, over the optimal time, a surveillance of packet delivery at the receivers, and a state observation for protocol convergence. It should be noted that there are two relevant convergence times. Prior to a full protocol convergence, i.e., an evacuation of the ETM state in all routers, packets may be already delivered on optimal paths. This convergence to optimal forwarding has been monitored separately at the receivers.

\subsection{Exploring the network topology space}

For a systematic approach to routing analysis, we first proceed in artificially exploring the topology space, i.e., the relative positions of the relevant network entities close to the moving source. The latter are given by the designated routers and the first intersection point $(\mathrm{X})$ of previous and next multicast tree. The degrees of freedom, which only depend on distance ratios, are covered by the two networks displayed in Fig. 4.

The simulation results for the two test networks as functions of intermediate router hops *DR-to-X, chosen between 0 and 30, are given in Fig. 5. As a striking outcome, test net 1 always delivers optimal forwarding without additional delays. This is due to the immediate discovery of the intersection router $\mathrm{X}$ by the EXTENDED FORWARDING ALGORITHM, which leads to packet delivery on the shortest path directly following a handover. Test net 2 admits similar performance values, but requires a re-routing, whenever the path lengths pDR-X equals nDR-X. This is reflected by a non-normal delay stretch and non-zero convergence time to optimal forwarding along a diagonal of the graph. Note that the characteristic of the delay stretch derives from the changing optimal forwarding times, while the absolute delay excess stems from one additional hop, i.e., nDR-X. In contrast,
Fig. 4 Test networks covering the relative routing topology in source vicinity

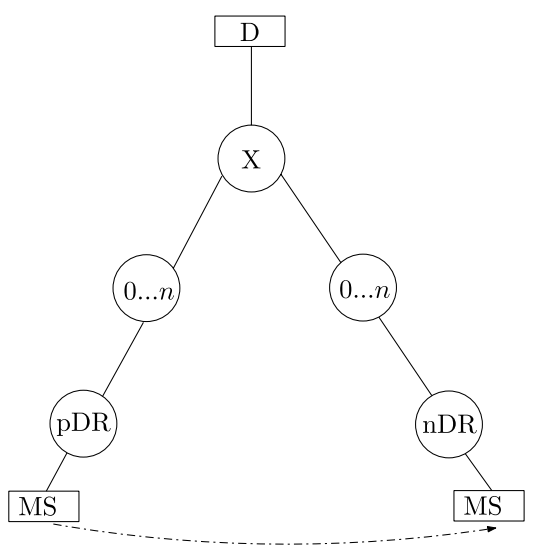

(a) Net 1

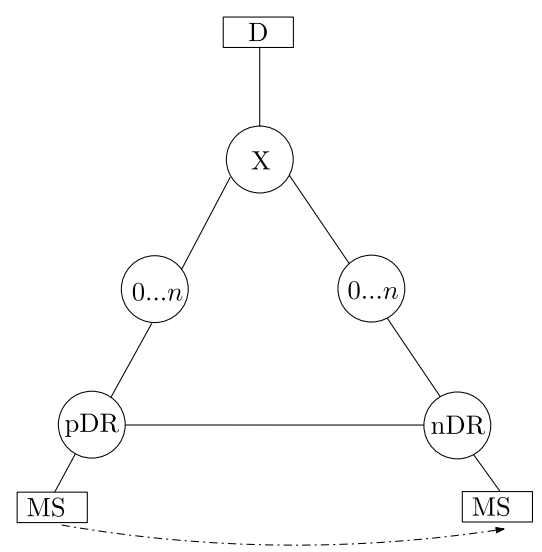

(b) Net 2 


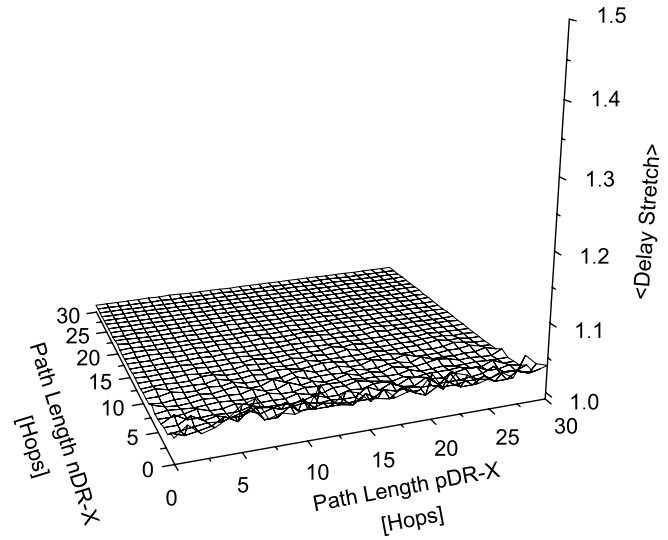

(a) Delay stretch net 1

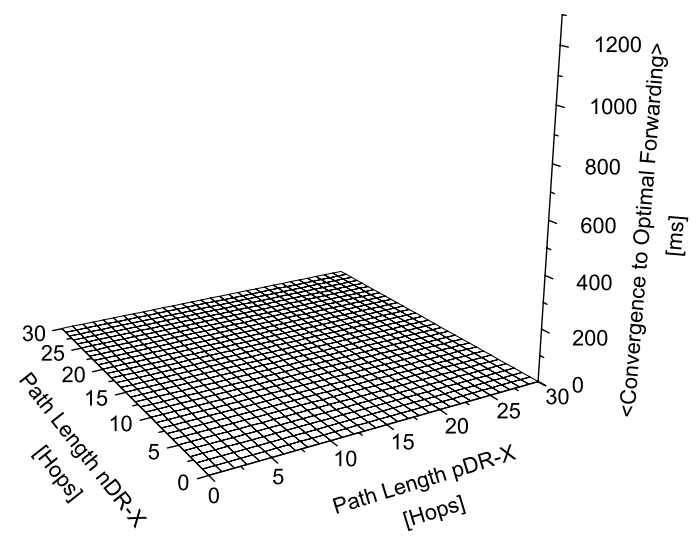

(c) Optimal forwarding net 1

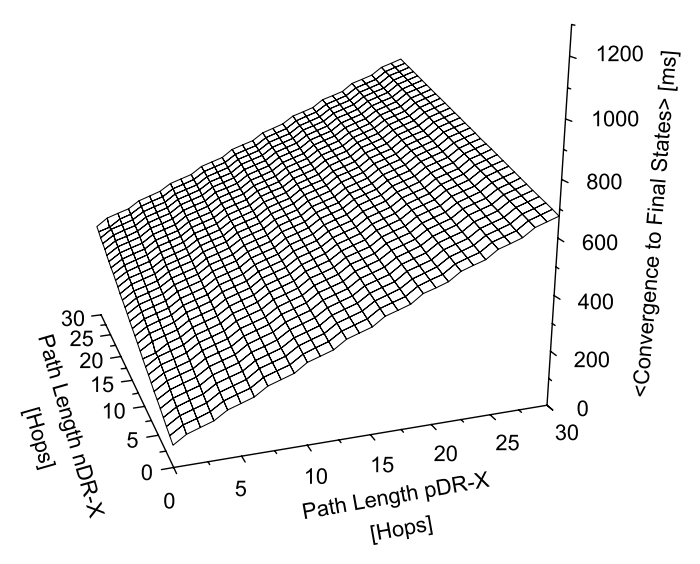

(e) Final convergence net 1

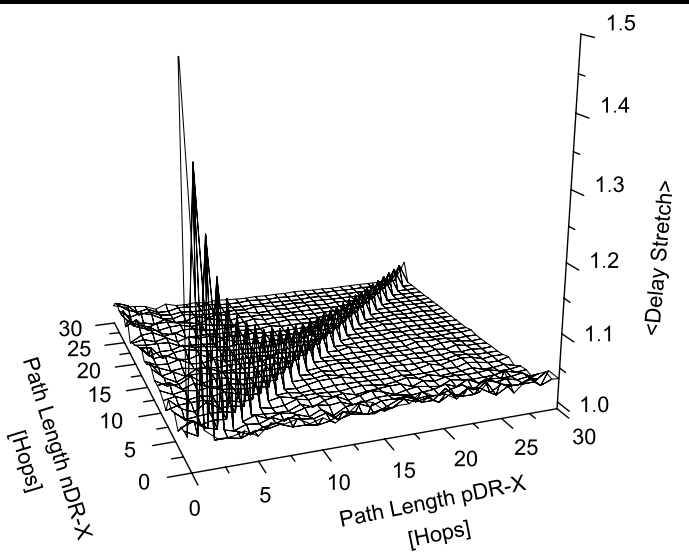

(b) Delay stretch net 2

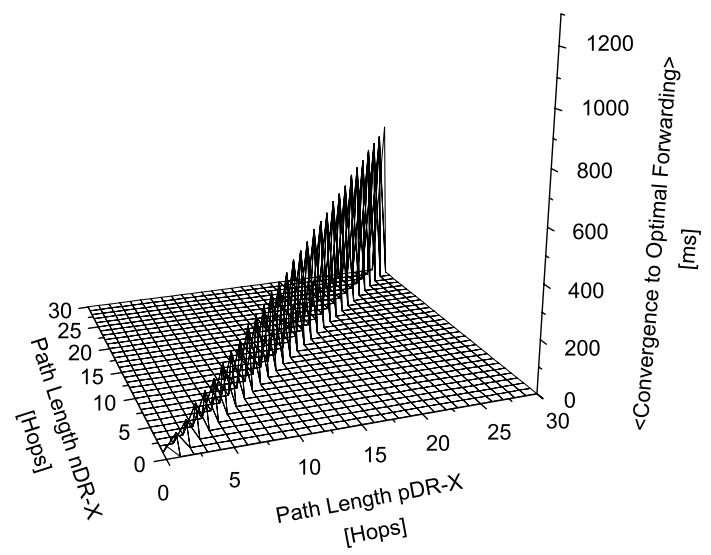

(d) Optimal forwarding net 2

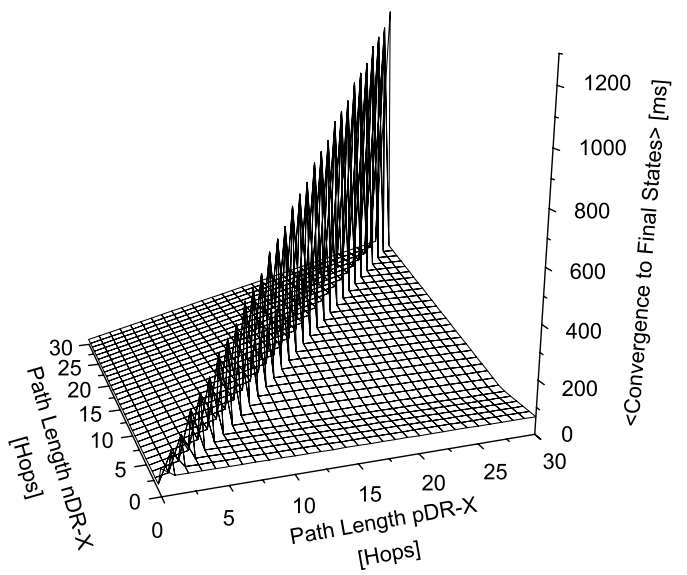

(f) Final convergence net 2

Fig. 5 Performance results for the test networks as functions of path lengths to the intersection X

routing convergence to final states is non-negligible in most cases. Routers along the path from $\mathrm{nDR}$ to $\mathrm{pDR}$ will change into ETM state. The routers on the previous tree, i.e., on the path from $\mathrm{X}$ to $\mathrm{pDR}$, will remain therein until a regular PIM prune is received. Forwarding states for test net 2 will be simply overwritten, when the distribution tree remains unaltered, i.e., $\operatorname{dist}(n D R, X) \neq \operatorname{dist}(p D R, X)$. A PIM prune towards pDR is required for $\operatorname{dist}(n D R, X)<\operatorname{dist}(p D R, X)$, while for $\operatorname{dist}(n D R, X)=\operatorname{dist}(p D R, X)$ the tree changes with the result of join and prune operations along the entire paths. No packet loss occurs in addition to layer 2 handoffs, why corresponding graphs are omitted. 
4.3 Single receivers within real-world topologies

In this section we present results for the same simulation experiments as described in the previous section, but performed on top of realistic topologies. Meshed networks obtained from real-world measurements on the one hand, a random variation of source and receiver positions on the other shall work out performance values that result from mixing protocol mechanisms. Mean performance results are shown in Fig. 6 as functions of pDR-nDR distance. ${ }^{3}$

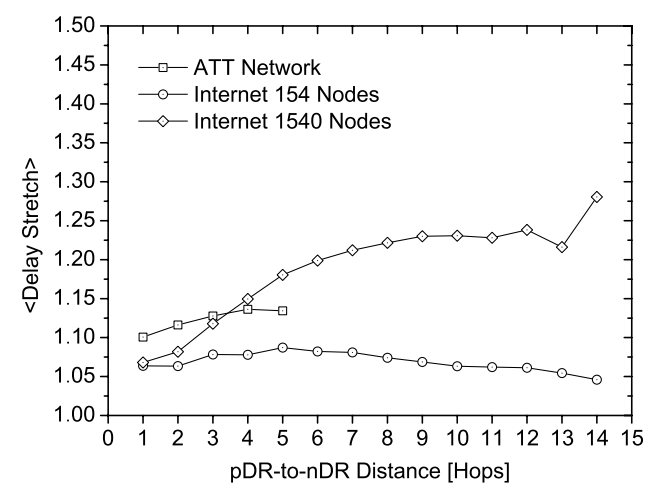

(a) Delay Stretch

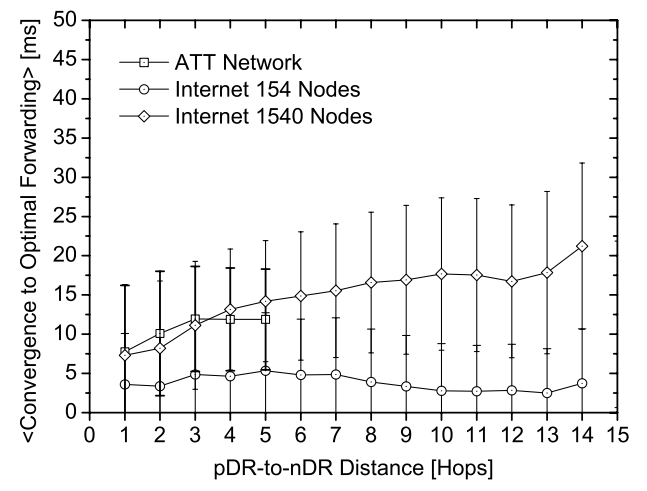

(b) Optimal Forwarding

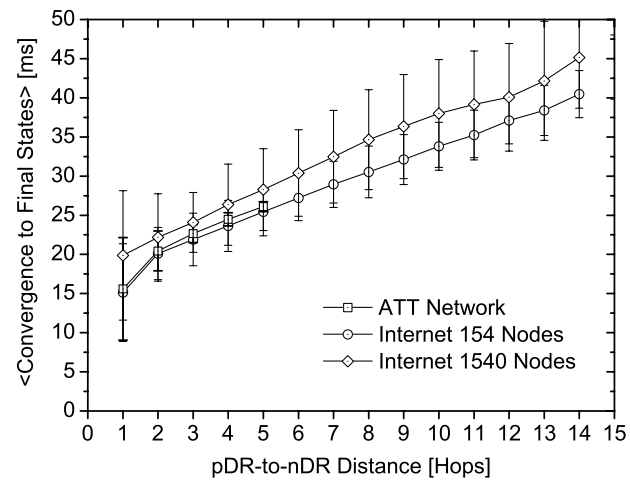

(c) Final Convergence

Fig. 6 Performance results for real-world topologies as functions of the mobility 'step size' (error bars show standard deviations)

\footnotetext{
${ }^{3}$ The access router distance, the mobility 'step size' in a figurative sense, can be regarded as a measure of complexity inherent to the prob-
}

Packet loss, which is only possible, when initial routing is guided along uneven triangles, did occur at a few events, only. Losses remain invisible in distributions, why the corresponding graphs are again not shown. The initial delay stretches for different topologies vary at an excess rate of about 5 to $25 \%$, only weakly dependent on access router distance. The convergence times to optimal forwarding remain bound by about $15 \mathrm{~ms}$. At our probe packet rate, the second datagram already travels on an optimal path.

Final convergence times clearly represent routing complexity as a function of mobility 'step size' and of topological diversity at comparably low values. Combined, the results indicate that the extremal values obtained for the artificial topologies equal out nicely when mixing in realistic topological settings. The overall performance degradation a receiver experiences under ETM multicast mobility management will thus be a delay of one packet that could have equally caused by regular jitter within the Internet.

\subsection{Multiple receivers within real-world topologies}

Simulations in our next scenario have been extended to include multiple receivers. Measurements of delay and forwarding time were taken individually for each receiver, allowing for differentiated evaluations. The results summarized in Fig. 7 are extracted for averages, minimal and maximal values taken event-wise from the sets of receivers. All values nicely reproduce the multicast nature of packet distribution in exhibiting almost no dependence on the number of receivers.

In detail, delay stretch values calculated for different topologies (see Figs. 7(a), (c), (e)) remain constant on average. Any multicast listener can thus expect an equal handover performance, independent of other joining parties. Identical to the single receiver scenario, measurements indicate delay variations of regular Internet scale. Min/max values naturally expand when taken over increasing receiver event sizes, but tend to saturate with enhanced exploration of the topology space by many receivers. Ranges between maximal and minimal delay stretch indicate absolute bounds on the performance variation of the ETM handover process. These bounds depend on the topological complexity of the underlying networks and thus vary with data sets. However, jitter and delay originating from multicast source mobility persistently remain within the range of seamless handover performance.

Similar results are obtained for convergence times to optimal forwarding (see Figs. 7(b), (d), (f)). On average, less than one packet is delivered on suboptimal paths. Larger,

lem [19]. Values range up to 14 in the Internet topology samples, while the maximum router distance within the ATT network is 5 . 
Fig. 7 Performance results for real-world topologies as functions of receiver multiplicities

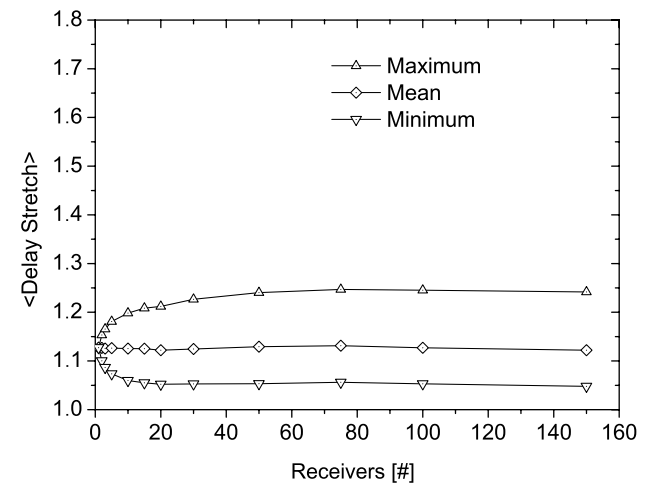

(a) Delay Stretch: ATT Network

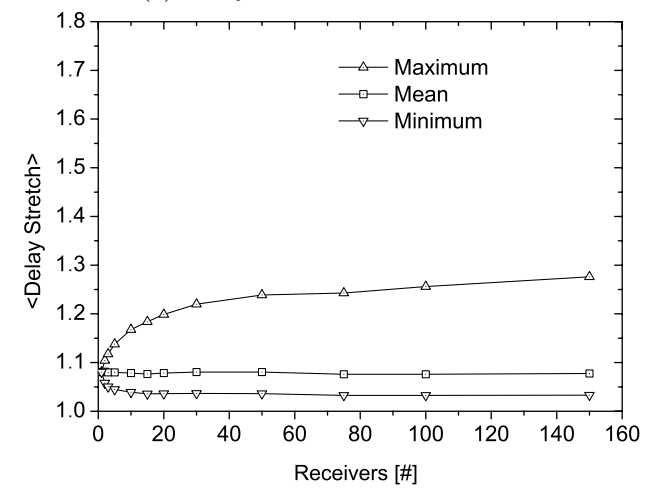

(c) Delay Stretch: Internet 154 Nodes

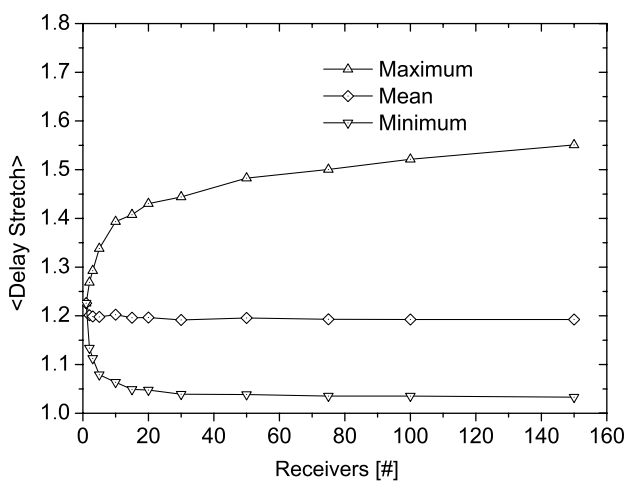

(e) Delay Stretch: Internet 1.540 Nodes

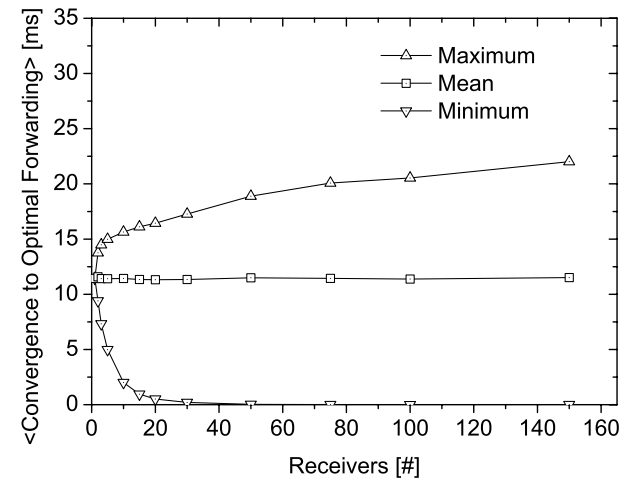

(b) Optimal Forwarding: ATT Network

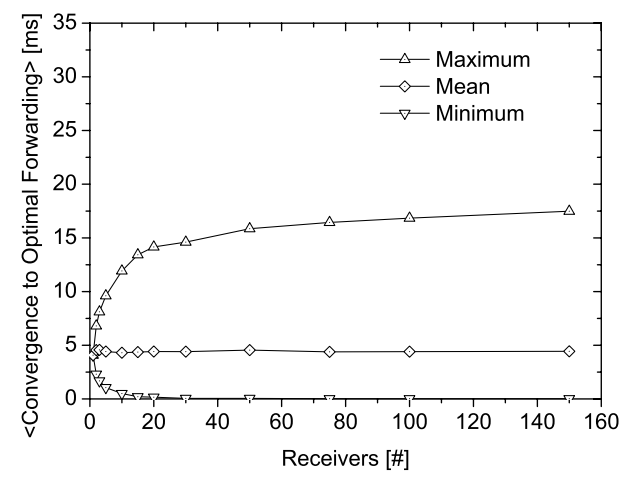

(d) Optimal Forwarding: Internet 154 Nodes

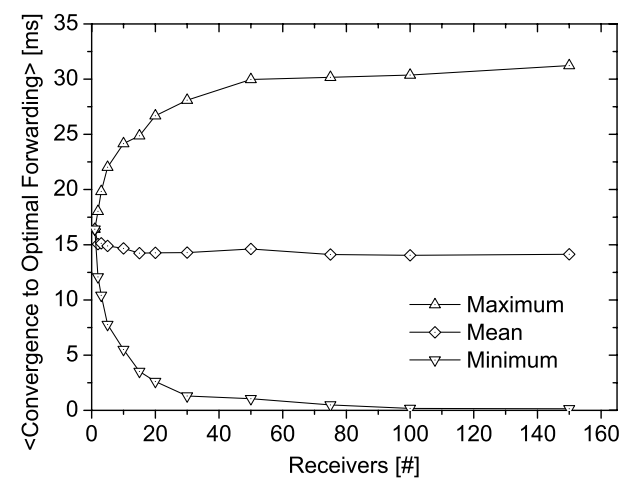

(f) Optimal Forwarding: Internet 1.540 Nodes

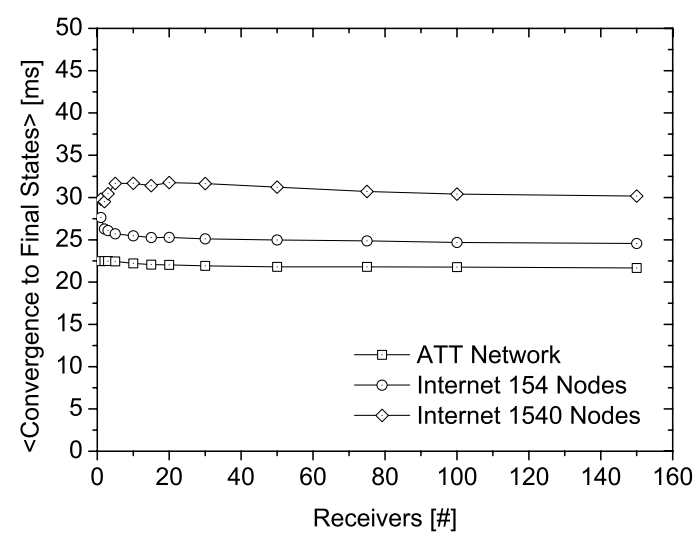

(g) Final Convergence 
more wide-span distribution trees for many receivers consistently approach a zero convergence-time minimum as a result of immediate shortcuts available at the source. Surprisingly, maximal convergence times quickly saturate at about 40 receivers, almost independent of the underlying topology. This observation indicates that protocol operations mainly remain local within the vicinity of the source and do inherit little influence of the overall network.

Final convergence times that are evaluated from all router states are displayed in Fig. 7(g). The constant curves clearly prove full scalability of the protocol with receiver multiplicities. In addition, results show little dependence on the topological data sets, thereby indicating the overall robustness of the ETM protocol.

\subsection{Multiple handovers}

In this final scenario, we investigate the protocol behavior under multiple rapid handovers. Three different speeds of the mobile source have been selected: $v=5 \mathrm{~km} / \mathrm{h}$ to represent a pedestrian, $v=20 \mathrm{~km} / \mathrm{h}$ for a cyclist, and a car at $v=80 \mathrm{~km} / \mathrm{h}$. Simulations were performed within the Internet topology of 1.540 core nodes.

The delay evolution for single event chains is visualized in Fig. 8 as a function of packet number. Handover events can be clearly identified as the vertical disturbances, while delays between handovers only fluctuate on a small scale. After each handover, delay measurements saturate at different absolute values due to varying topological conditions. A detailed performance evolution can be extracted from the inserts. Following a period of complete packet loss due to layer 2 handoff and MIPv6 reconfiguration, short delay peaks occur that rapidly fade. These small peaks-resulting from one or two delayed packets-are the only visible effect of the ETM protocol. In particular, protocol performance remains completely independent of handover iterations and speed, as the protocol operates on a significantly smaller timescale than physical source movement.

Further insight on protocol convergence under rapid movement becomes apparent from monitoring individual router states. Figure 9 displays the relative number of router states as a function of continuous simulation time for single event chains. Values are measured by evaluating the cumulative total number of states divided by the total number of routers involved. Again, handover events can be clearly identified at vertical lines, resulting from a router state enhancement that quickly diminishes. Horizontal lines indicate the state multiplicities after protocol convergence. As a striking outcome, the protocol always convergences well before handover iterations, even at the fast movement of cars.

Details of the state evolution at handovers become visible from the inserts. The raise and fall of router state multiplicities nicely appear as smooth "bumps" on a very finegranular scale. These values are the result of mixing state

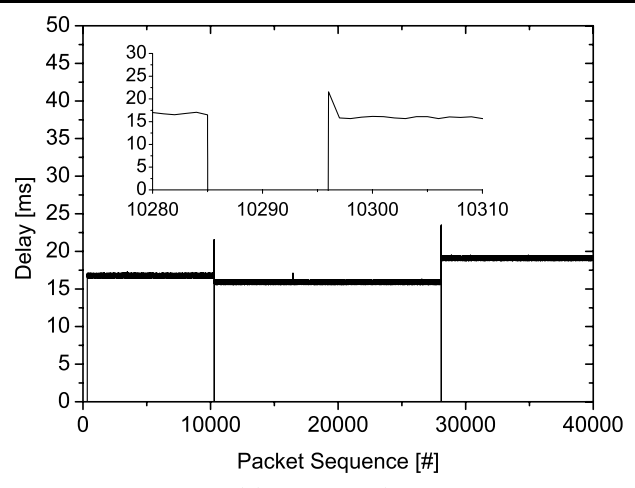

(a) $v=5 \mathrm{~km} / \mathrm{h}$

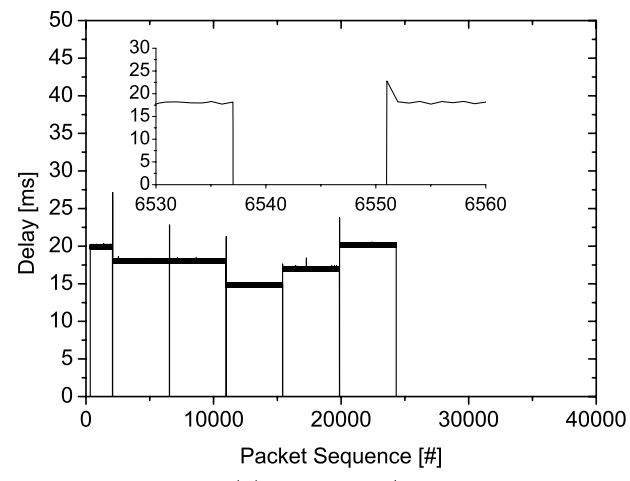

(b) $v=20 \mathrm{~km} / \mathrm{h}$

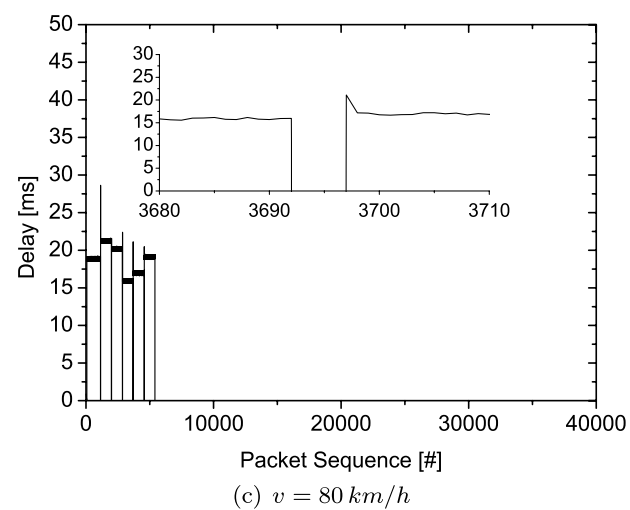

Fig. 8 Delay for multiple handovers at different speeds $v$ of a mobile source

creation and erasure within the network, occurring in close temporal overlap, so that the overall period of state fluctuation remains within a period of less than 50 milliseconds. These results not only grant a close view on the rapid, highly parallel protocol convergence process, observed under the assumption of link delays typical for geographic proximity, but also show that the ETM protocol is suitable for source movement at all speeds common to our mobile world.

\section{Combining mobility optimizations and enhanced tree morphing}

The Enhanced Tree Morphing protocol as presented and analyzed in the previous sections can be transparently applied 


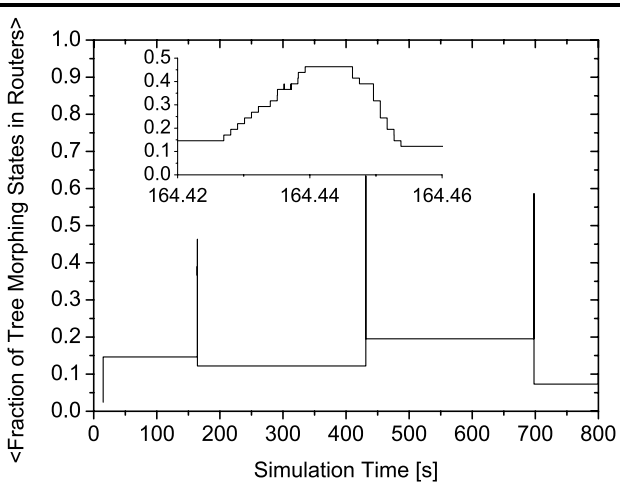

(a) $v=5 \mathrm{~km} / \mathrm{h}$

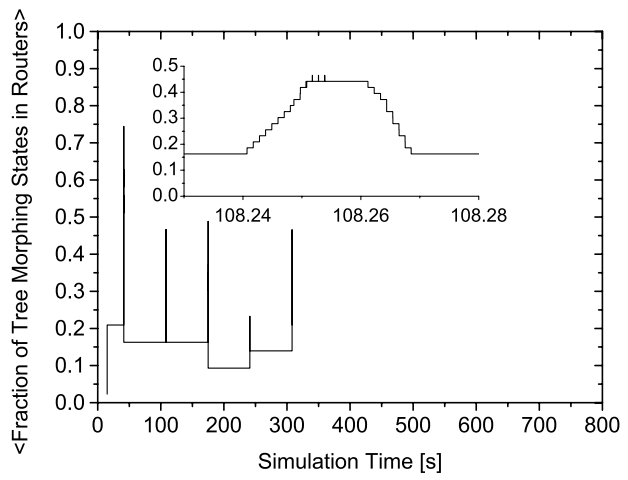

(b) $v=20 \mathrm{~km} / \mathrm{h}$

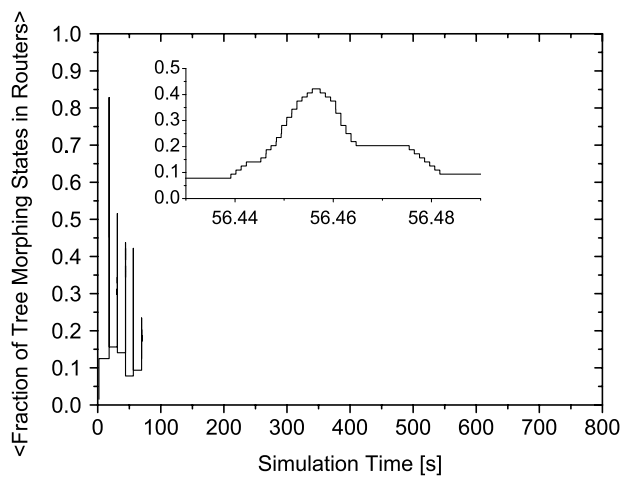

(c) $v=80 \mathrm{~km} / \mathrm{h}$

Fig. 9 Relative portion of tree morphing states per multicast forwarder for multiple handovers and different speeds $v$ of a mobile source

in the presence of current mobility optimization protocols such as FMIPv6 [21], HMIPv6 [22], or in multihomed environments. ${ }^{4}$ Nevertheless, particular advantages can be taken from individual mobility management schemes, which will be introduced in this section.

\footnotetext{
${ }^{4}$ PMIPv6 [29] has not been designed as an end-to-end protocol with route optimization, but shields mobility of a node by tunneling to a fixed Local Mobility Anchor. Multicast source mobility remains transparent, but adaptive routing unfeasible.
}

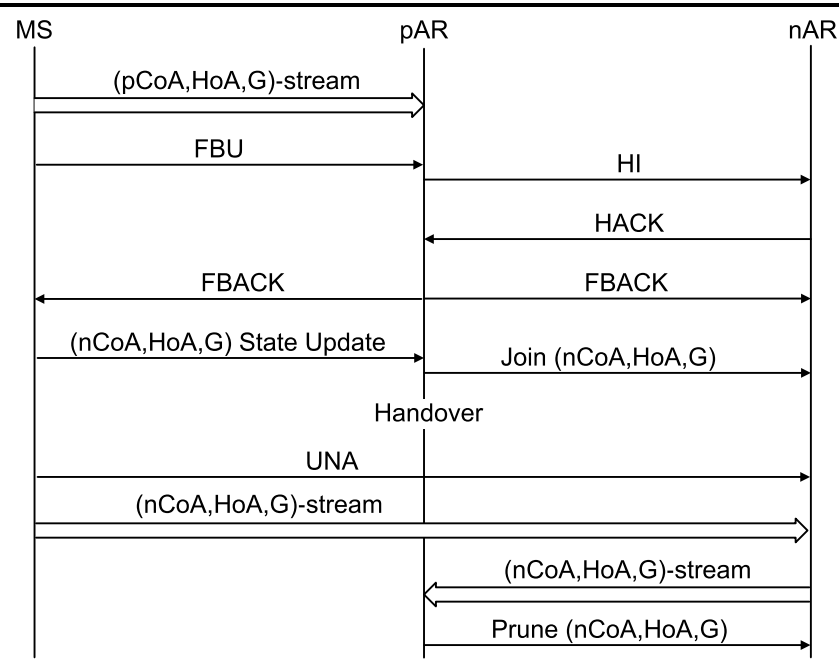

Fig. 10 Flowchart of joint FMIPv6 and ETM signaling

\subsection{Fast mobile IPv6}

Fast Mobile IPv6 has been designed to hide latencies of IP reconfiguration and binding updates, and can operate in a predictive and a reactive mode. FMIPv6 handover assistance is obtained from access routers that mirror the role of $\mathrm{pDR}$ and nDR in the Tree Morphing approach. This reverse symmetry can be exploited to simplify ETM operations as follows.

In predictive mode, a mobile source anticipates a handover, and receives its new CoA as part of a Fast Binding Acknowledgement (FBACK) prior to disconnecting. Still at its previous attachment, MS adds multicast state update headers to its traffic flow as presented in Fig. 3. In response, the previous access router (pAR) issues an $(n C o A, H o A, G)$ join to the interface of the tunnel that has been established with the new access router (nAR) as part of regular FMIPv6 operations. While the mobile node changes location, new ( $n C o A, H o A, G)$ states are established on the previous distribution tree, and the ETM tree optimization will initiate the creation of a new shortest path tree. At its new point of attachment, the MS continues multicast transmission using its new Care of Address. Data will be distributed to the newly established (partial) tree, and-via the nAR-topAR reverse tunnel- to the previous tree, until old branches are removed, and pAR prunes its tunnel interface. The flow chart of FMIPv6 multicast extensions is displayed in Fig. 10.

In reactive mode, a mobile source uses the reverse tunnel established with pAR for updating states of the previous distribution tree. After reconnecting to the new network, MS submits multicast data with state update headers (see Fig. 3) to the reverse tunnel, and thereafter proceeds in native multicast transmission exactly as in the predictive mode.

The joint application of FMIPv6 and ETM not only reduces multicast state update signaling cost, but in its pre- 
dictive mode allows for tree optimization in parallel with layer 2 handoff. Like for unicast, this will hide layer 2 latencies and - at opportune conditions - may lead to optimal multicast routing at the time the MS reconnects.

\subsection{Hierarchical mobile IPv6}

Hierarchical Mobile IPv6 maintains handover transparency throughout a micro-mobile domain of a Mobility Anchor Point (MAP). HMIPv6 regional tunneling preserves the shielding of micro mobility in the presence of ETM. InterMAP handovers for mobile multicast sources can be performed just by regular ETM signaling, while HMIPv6 Regional CoAs replace the conventional Care of Addresses. In this sense, operations of the ETM protocol remain fully transparent in the presence of HMIPv6.

\subsection{Multihoming}

A mobile node may maintain multiple points of attachment in different scenarios [30], the major objective being an ubiquitous, permanent and fault-tolerant access to the Internet. In the situation of a handover, the characteristic advantage of multihomed connectivity is an interface-wise iterative change of attachment. Such a "make-before-break" approach can be integrated with the ETM protocol in the following way.

A multihomed mobile source that issues multicast traffic via a single interface may experience a handoff at any point of attachment. If the multicast source interface remains connected, it simply continues service. Otherwise, and under the assumption that another interface is equally suitable for multicast service, it may attempt to smooth the handoff by immediately switching its source interface and performing regular state updates as described in Sect. 3. Whenever a handoff can be anticipated, the unicast state update may be issued in advance, while data continues to flow from the previous point of attachment.

\section{Conclusions and outlook}

In this article, the Enhanced Tree Morphing protocol was introduced and extensively evaluated. ETM provides address duality management for SSM senders and seamlessly performs handover operations for source mobility. Designed as an optimized improvement of the previously developed Tree Morphing, it solely relies on lightweight state update messages composed of standard IPv6 headers. Signaling is limited to one unicast message between designated routers, and extension headers added to the first data packet(s) subsequent to handover. Cryptographically strong update authentication is inherited from the AuthoCast framework. These optimizations significantly advanced the protocol performance, as could be confirmed by a thorough protocol evaluation.

In extensive simulations, we systematically explored topological routing constellations. It could be revealed that only a narrow fraction of the state space causes significant re-routing, while the majority of constellations lead to immediate optimal packet forwarding and rapid protocol convergence. Under mixing conditions of real-world topological data, ETM delivers close to optimal performance. Packet loss and re-ordering remain statistically absent, while the characteristic handover performance causes a delay stretch of about 10 to $20 \%$ for the first packet only. Protocol convergence approved strictly compliant with rapid motion. Following a handover, packets are delivered on optimal paths after about $15 \mathrm{~ms}, 30 \mathrm{~ms}$ are required on average for final convergence. Results showed to be fairly independent of receiver numbers and topologies. Even a fast moving car browsing through an 802.11 WLAN regime will experience handoffs at a rate of seconds. To the best of our knowledge, ETM is the first protocol that provides robust multicast route optimization at real-time mobility conditions.

Future work shall expose ETM routing to reality and explore its practical feasibility. We hope for seeing live video transmissions on IPTV sport channels that originate from a lightweight source moving rapidly in the field.

Acknowledgements This work has been supported in part by the German Bundesministerium für Bildung und Forschung within the project Moviecast (http://moviecast.realmv6.org).

\section{References}

1. Johnson, D. B., Perkins, C., \& Arkko, J. (2004). Mobility support in IPv6. RFC 3775, IETF.

2. Deering, S. E. (1989). Host extensions for IP multicasting. RFC 1112, IETF.

3. Schmidt, T. C., Wählisch, M., \& Fairhurst, G. (2008). Multicast mobility in MIPv6: problem statement and brief survey. IRTF internet draft, work in progress 05, MobOpts.

4. Holbrook, H., \& Cain, B. (2006). Source-specific multicast for IP. RFC 4607, IETF.

5. Rosenberg, J., Schulzrinne, H., Camarillo, G., Johnston, A., Peterson, J., Sparks, R., Handley, M., \& Schooler, E. (2002). SIP: session initiation protocol. RFC 3261, IETF.

6. Schmidt, T. C., \& Wählisch, M. (2008). Group conference management with SIP. In S. Ahson \& M. Ilyas (Eds.), SIP handbook: services, technologies, and security (pp. 123-158). Boca Raton: CRC Press.

7. Perkins, C. E., \& Johnson, D. B. (1996). Mobility support in IPv6. In MobiCom '96: Proceedings of the 2nd annual international conference on mobile computing and networking (pp. 2737). New York: ACM Press. 
8. Xylomenos, G., \& Polyzos, G. C. (1997). IP multicast for mobile hosts. IEEE Communications Magazine, 35(1), 54-58.

9. Romdhani, I., Bettahar, H., \& Bouabdallah, A. (2006). Transparent handover for mobile multicast sources. In P. Lorenz \& P. Dini (Eds.), Proceedings of the IEEE ICN'06. New York: IEEE Press.

10. Fenner, B., Handley, M., Holbrook, H., \& Kouvelas, I. (2006). Protocol independent multicast—sparse mode (PIM-SM): protocol specification (Revised). RFC 4601, IETF.

11. Thaler, D. (2001). Supporting mobile SSM sources for IPv6. Proceedings of IETF meeting, individual.

12. Jelger, C., \& Noel, T. (2002). Supporting mobile SSM sources for IPv6 (MSSMSv6). Internet draft, work in progress (expired) 00, individual.

13. Wählisch, M., \& Schmidt, T. C. (2007). Exploring the routing complexity of mobile multicast-a semi-empirical study. In CoNEXT '07: Proceedings of the 2007 ACM CoNEXT conference, Columbia University (pp. 1-2). New York: ACM Press.

14. Chang, R. S., \& Yen, Y. S. (2004). A multicast routing protocol with dynamic tree adjustment for mobile IPv6. Journal of Information Science and Engineering, 20, 1109-1124.

15. Vida, R., Costa, L., \& Fdida, S. (2002). M-HBH-efficient mobility management in multicast. In Proceedings of NGC '02 (pp. 105-112). New York: ACM Press.

16. Costa, L. H. M. K., Fdida, S., \& Duarte, O. (2001). Hop by hop multicast routing protocol. In SIGCOMM '01: Proceedings of the 2001 conference on applications, technologies, architectures, and protocols for computer communications (pp. 249-259). New York: ACM Press.

17. O'Neill, A. (2002). Mobility management and IP multicast. Internet draft, work in progress (expired) 01, IETF.

18. Schmidt, T. C., \& Wählisch, M. (2005). Extending SSM to MIPv6-problems, solutions and improvements. Computational Methods in Science and Technology, 11(2), 147-152. Selected Papers from TERENA Networking Conference, Poznań, May 2005.

19. Schmidt, T. C., \& Wählisch, M. (2006). Morphing distribution trees - on the evolution of multicast states under mobility and an adaptive routing scheme for mobile SSM sources. Telecommunication Systems, 33(1-3), 131-154.

20. Lee, H., Han, S., \& Hong, J. (2006). Efficient mechanism for source mobility in source specific multicast. In K. Kawahara \& I. Chong (Eds.), LNCS: Vol. 3961. Proceedings of ICOIN2006 (pp. 82-91). Berlin, Heidelberg: Springer.

21. Koodli, R. (2008). Mobile IPv6 fast handovers. RFC 5268, IETF.

22. Soliman, H., Castelluccia, C., Malki, K., \& Bellier, L. (2008). Hierarchical mobile IPv6 (HMIPv6) mobility management. RFC 5380, IETF.

23. Schmidt, T. C., Wählisch, M., Christ, O., \& Hege, G. (2008). AuthoCast-a mobility-compliant protocol framework for multicast sender authentication. Security and Communication Networks, 1(6), 495-509.

24. Partridge, C., \& Jackson, A. (1999). IPv6 router alert option. RFC 2711, IETF.

25. Arkko, J., Vogt, C., \& Haddad, W. (2007). Enhanced route optimization for mobile IPv6. RFC 4866, IETF.

26. Varga, A. et al. (2007). The OMNeT++ discrete event simulation system. http://www.omnetpp.org.

27. Heckmann, O., Piringer, M., Schmitt, J., \& Steinmetz, R. (2003). On realistic network topologies for simulation. In MoMeTools '03: Proceedings of the ACM SIGCOMM workshop on models, methods and tools for reproducible network research (pp. 28-32). New York: ACM Press.

28. Govindan, R., \& Tangmunarunkit, H. (2000). Heuristics for internet map discovery. In Proceedings IEEE INFOCOM 2000 (Vol. 3 , pp. 1371-1380). Piscataway: IEEE Press.
29. Gundavelli, S., Leung, K., Devarapalli, V., Chowdhury, K., \& Patil, B. (2008). Proxy mobile IPv6. RFC 5213, IETF.

30. Montavont, N., Wakikawa, R., Ernst, T., Ng, C., \& Kuladinithi, K. (2008). Analysis of multihoming in mobile IPv6. Internet-draft, work in progress 05 , IETF.

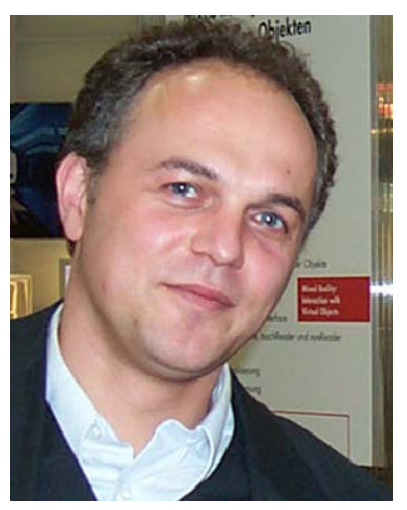

Thomas C. Schmidt is professor of Computer Networks \& Internet Technologies at Hamburg University of Applied Sciences (HAW) and leads the Internet Technologies research group (INET) there. Prior to moving to Hamburg, he headed the computer centre of FHTW Berlin for many years, and continued work as an independent project manager later. He studied mathematics and physics at Freie Universität Berlin and University of Maryland. He has continuously conducted numerous national and international projects. His current interests lie in next generation Internet (IPv6), mobile multicast and multimedia networking, as well as XML-based hypermedia information processing. He serves as co-editor and technical expert in many occasions and is actively involved in the work of IETF.

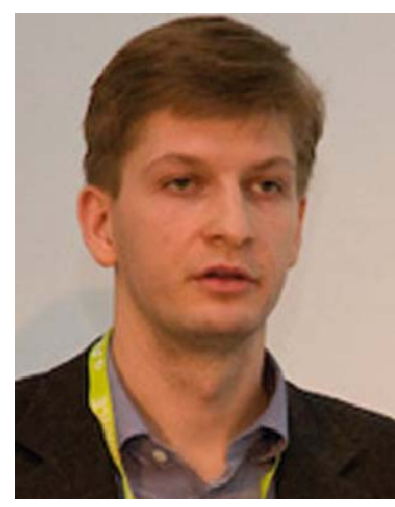

Matthias Wählisch studied computer science and contemporary German literature at Freie Universität Berlin, where he completed his diploma thesis on structured hybrid multicast routing. He continues his research at the Computer Systems $\&$ Telematics group there, and is also with the INET research team at HAW Hamburg. He started professional activities at the networking group of the computer centre of FHTW Berlin while at high school. $\mathrm{He}$ is the co-founder of link-lab, a start-up company in the field of next generation networking. His major fields of interest lie in the design and analysis of networking protocols, with a special focus on mobility and group communication in underlay and overlay, where he looks back on ten years of professional experience in project work and publication.

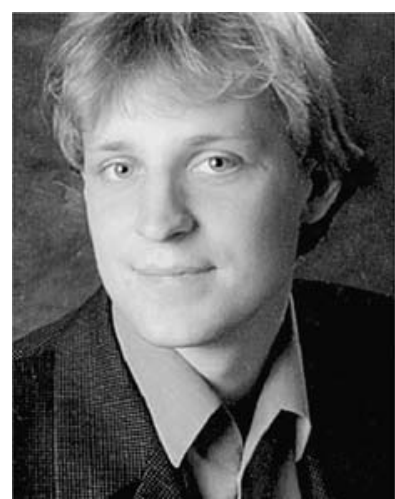

Maik Wodarz is a member of the INET scientific team at Hamburg University of Applied Sciences. He studied computer science at Technische Universität Berlin, and graduated with a work about a WLAN tracer for wireless fieldbus systems. His major field of interest lies in the analysis and simulation of computer networks. 